\title{
El Paciente Respirador Bucal: Manifestaciones Clínicas. Evaluación de signos y síntomas.
}

Oral Respirator Patient: Clinical Manifestations. Evaluation signs and symptoms.

Fecha de Recepción

21 de junio de 2012
Paciente Respirador Bucal:Manifestações clínicas. Avaliação Sinais e sintomas.
Silvia Raquel Gonzalez de Saglio

Jefe de Trabajos Prácticos. Cátedra

Fundamentos de la Ortodoncia

Carmen ltati Collante de Benitez

Profesora Adjunta a Cargo Cátedra

Fundamentos de la Ortodoncia
Aceptado para su publicación

09 de octubre de 2012

\section{Resumen}

El paciente respirador bucal, presenta un conjunto de signos y síntomas como manifestación de la patología respiratoria ocasionada por distintas causas, (obstructivas anatómicas funcionales o por hábitos), que actuando en forma prolongada, dificultan la entrada de aire por la nariz, obligando al niño a abrir la boca para hacerlo, priorizándose la función vital.

Esta disfunción respiratoria, ocasiona problemas que afectan varias partes del organismo, con repercusiones de orden local y general.

La correcta observación, permite distinguir los signos y síntomas, más comunes que son importantes para facilitar el diagnóstico, elegir el procedimiento terapéutico en forma precoz y adecuada; como también, determinar la necesidad de realizar interconsultas con otras especialidades, para que el paciente reciba tratamiento temprano e integral.

\section{Palabras Claves}

Síndrome - Respirador bucal - Respirador Nasal.

\section{Summary}

The mouth breather patient presents a set of signs and symptoms as a manifestation of the respiratory pathology caused by different causes (obstructive anatomic, functional or habits), which acts prolonged complicate the entry of air through the nose, forcing the child to open his mouth to do, prioritizing the vital function

This respiratory dysfunction, causes prob- 
lems affecting various parts of the body, with repercussions of a local and general

The correct observation allows us to recognize the signs and symptoms, most common that are important to facilitate diagnosis, choose early and appropriate therapeutic procedure and also determine the need for consultations with other disciplines so that the patient receives comprehensive treatment.

\section{Keywords}

Syndrome - nasal respirator - Oral respirator.

\section{Resumo}

O paciente respirador bucal apresenta um conjunto de sinais e sintomas como uma manifestação da patologia respiratória causada por diferentes causas (obstrutiva hábitos anatômicas, funcionais ou), que atua prolongou complicar a entrada de ar através do nariz, forçando a criança para abrir a boca para fazer, prioriza

Esta disfunção respiratória, causa problemas que afetam várias partes do corpo, com repercussões de um local e geralndo a função vital.

A observação correta nos permite reconhecer os sinais e sintomas, mais comuns que são importantes para facilitar o diagnóstico, escolha precoce e adequado procedimento terapêutico e também determinar a necessidade de consultas com outras disciplinas para que $\circ$ paciente recebe tratamento integral.

\section{Palavras chave}

Síndrome - Respirador bucal - Respirador Nasal.

\section{Introducción}

El Síndrome del Respirador Bucal, es el conjunto de signos y síntomas presentes ante la alteración patológica de la función nasorrespiratoria. Si existen dificultades para que la función respiratoria se lleve acabo por la nariz, se produce su reemplazo por la respiración bucal. Las causas más frecuente de respiración bucal sustitutiva, son hipertrofia de adenoides, desviación de tabique, pólipos, rinitis, alergias, obstrucciones faringeas por hipertrofia de amígdalas palatinas. Pero también existen respiradores bucales sin causa aparente que la provoque, lo que se considera que es un hábito'.
Para diagnosticar y tratar los problemas de respiración bucal, se deben reconocer las distintas manifestaciones presentes, siendo para ello fundamental comprender y conocer la anatomía y fisiología normal del sistema respiratorio. El aire pasa por la nariz y los senos paranasales, antes de entrar a la rinofaringe a través de las coanas. Existen siete senos paranasales: dos maxilares, dos frontales, dos etmoidales, y uno esfenoidal. La rinofaringe, donde el aire llega después de pasar por la nariz y los senos paranasales forma parte de la faringe, la cual desempeña funciones respiratorias $y$ alimentarias.

La faringe va desde la base del cráneo hasta la sexta vértebra cervical, donde se une al esófago y a la laringe. Está situada en la parte posterior de la cavidad nasal, cavidad bucal y laringe. Por esta razón está dividida en tres partes:

la superior, también llamada nasal o rinofaringe.cavum o porción media, también llamada bucal u orofaríngea;

la parte inferior, también llamada laringea, hipofaringe $o$ laringofaringe

La regulación neuronal de la respiración se produce en el tronco encefálico, en la sustancia reticular y la porción baja del puente. Las señales nerviosas son trasmitidas hacia los músculos de la respiración: diafragma (inspiración) y abdominales (expiración) ${ }^{2}$.

Durante la respiración nasal es necesario que la boca se cierre en algún punto. Normalmente esto se da por el sellamiento labial. Pero este cierre también puede ocurrir en la porción media, con el dorso de la lengua en contacto con el paladar duro y también posteriormente con la base de la lengua en el paladar blando. En caso de no haber sellamiento en ninguno de estos puntos se presentará respiración bucal o mixta, es decir por la nariz y por la boca.

Las manifestaciones del paciente respirador bucal, pueden observarse en individuos a temprana edad con problemas respiratorios iniciales, donde la terapia preventiva será la adecuada, aunque también en pacientes adolescentes 0 adultos donde las alteraciones serán más graves y de mayor magnitud, a veces irreversibles sobre todo cuando al problema funcional se asocie la predisposición genética para su desarrollo ${ }^{3}$. 


\section{Manifestaciones Clínicas}

Las manifestaciones clínicas puedan presentarse en individuos con cambios locales $y$ generales.

\section{Cambios locales}

Entre los cambios locales que se manifiestan en el respirador bucal, se encuentran las alteraciones faciales y bucales.

Cuando el problema respiratorio se debe a obstrucciones de las vías respiratorias altas o nasofaringe, observaremos facie adenoidea, (Figura I) caracterizada por presentar los labios separados, el cierre bucal se realiza con esfuerzo con marcada contractura de los músculos del mentón.

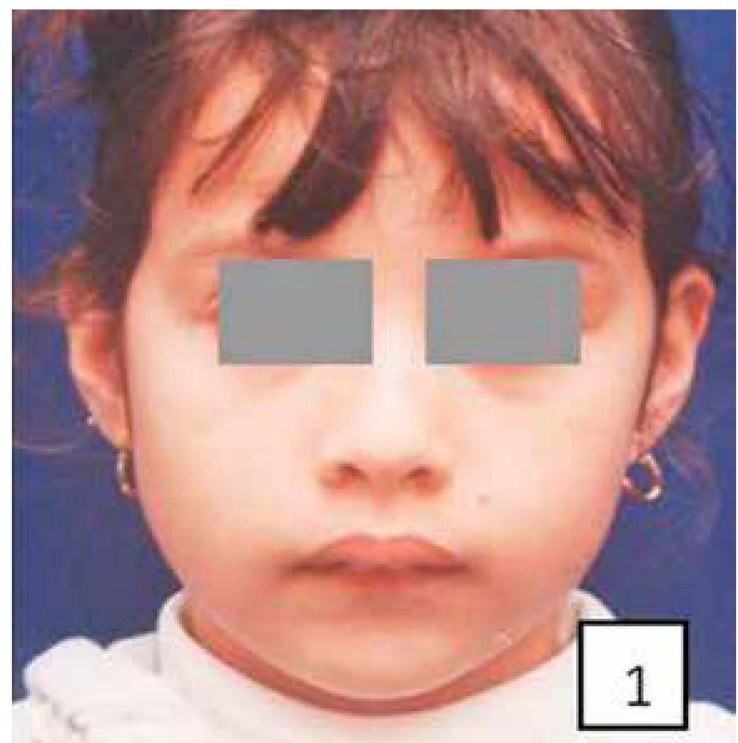

Figura I

El cierre bilabial es el efecto de cerrar y mantener cerrados los labios, y constituye la señal fundamental que permite determinar clínicamente la normalidad o alteración de la tonicidad de los labios; estos además forman parte primordial del mecanismo del buccinador, permitiendo el equilibrio vestibulolingual que mantiene los dientes en posición y que si se rompe produce incompetencia bilabial ${ }^{4}$. Proffit, plantea que al realizar el examen facial, los labios morfológicamente adecuados deben tocarse levemente $y$ efectual el cierre bucal cuando la mandíbula este en reposo. Si los labios quedan separados entre tres milímetros o más, podemos decir que estamos en presencia de una incompetencia bilabial.
Pueden exponerse las piezas dentarias del sector anterosuperior, debido a que el labio superior es corto hipotónico y no s alcanza a cubrir las piezas dentarias. Así lo refiere Roth y cols., planteando que los labios relajados permiten observar solo $3 \mathrm{~mm}$. de los incisivos centrales por debajo del bermellón del labio.

El labio inferior se presenta evertido, hipertónico e interpuesto entre ambas arcadas dentarias, ubicándose por palatino de las piezas dentarias antero superiores a las que presiona con fuerza vestibulizadora (Figura 7). Ambos labios pueden estar agrietados. El tercio inferior de la cara puede estar aumentado por el crecimiento divergente de las basales. Las narinas se presentan estrechas por falta de función de los múscu-

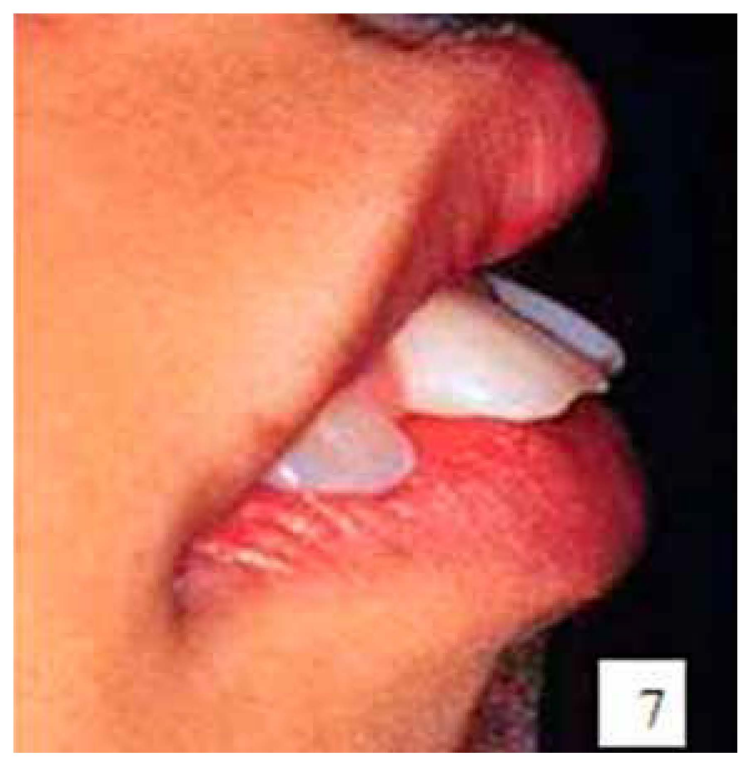

Figura 7

los alares. El aspecto facial es pálido, ojeroso con ojos cansados y sin brillo, debido a que todo el organismo y sus funciones van a estar alterados por el insuficiente aporte de oxígeno ${ }^{5}$.

En el perfil facial, (Figura 2) al trazar el plano facial sobre los tejidos blandos, (glabelar- pogonio) se observa marcada retroposición mandibular por desplazamiento del Po por distal del plano. Si trazamos el plano Estético de Riketts, se verá que ambos labios se acercan o sobrepasan anteriormente al plano, siendo el labio superior el más adelantado por la marcada protrusión dentoalveolar.

En cuanto a las alteraciones bucales, La característica bucal más destacada es la protrusión 


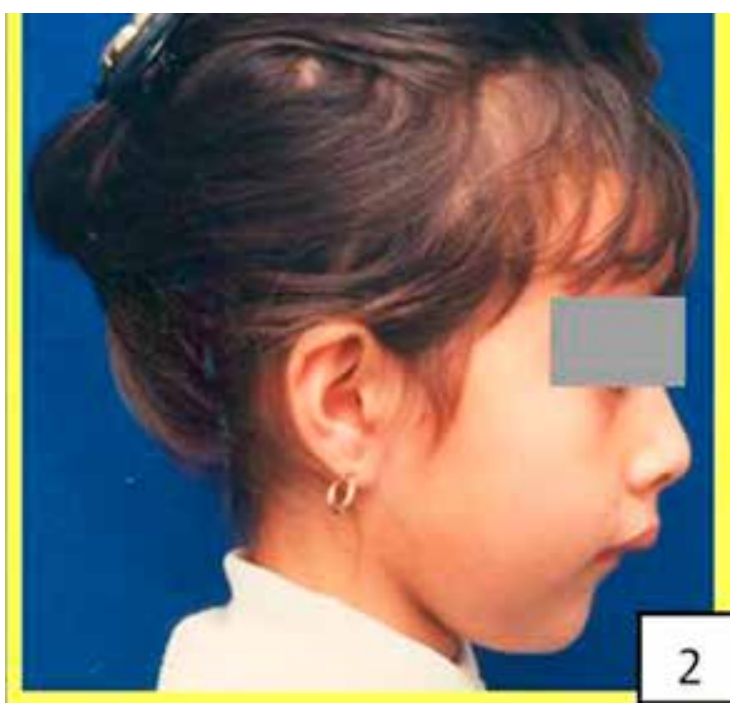

Figura 2

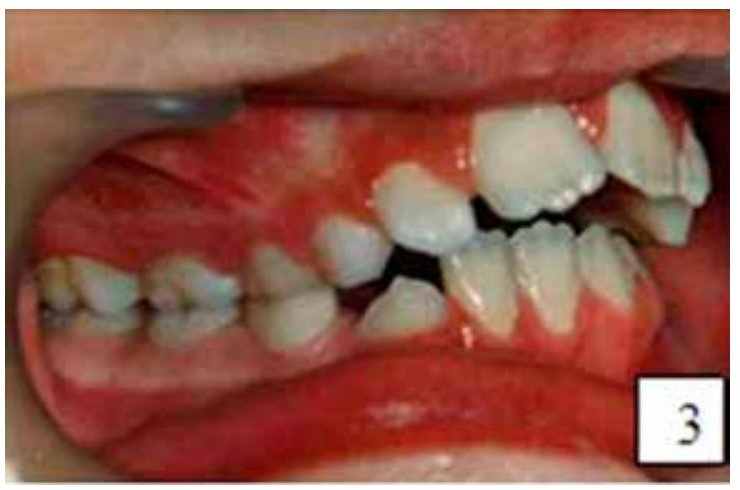

Figura 3

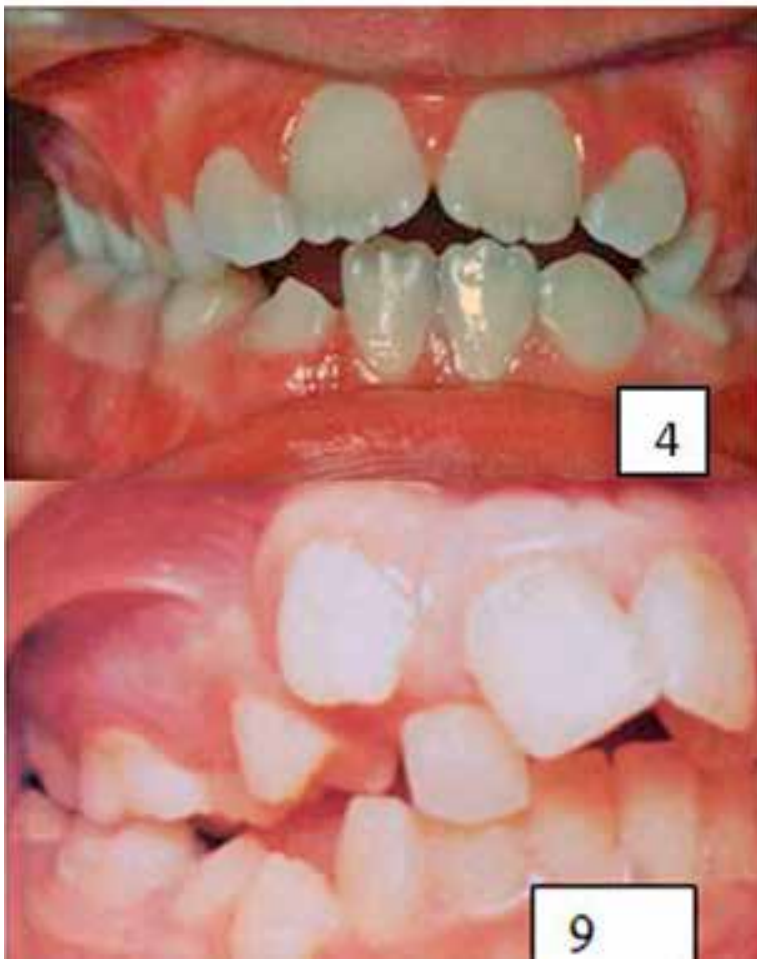

dentoalveolar superior y el retrognatismo inferior. Resalte aumentado, presentando el paciente Clase II I Ia de Angle. (Figura 3) ${ }^{6}$.

En lo que respecta al crecimiento del maxilar superior, la posición normal de la lengua adosada a la bóveda palatina va a estimular el correcto desarrollo transversal del paladar y por lo tanto del piso de las fosas nasales, por lo que la lengua constituye un factor importante en el crecimiento y desarrollo del maxilar superior. En el respirador bucal la lengua adopta una posición baja, para dejar via libre a la entrada de aire, y porque la presión que ocasiona el paso del aire, la hace descender aún más. Como consecuencia directa, el estimulo lingual necesario para el desarrollo de los maxilares desaparece, creciendo estos en sentido vertical y no en sentido anteroposterior. Tanto el paladar como los procesos alveolares, son las estructuras directamente relacionadas con la función lingual y va a repercutir en mayor o menor grado en el desarrollo esquelético peribucal, originando diversos grados de dismorfosis a nivel de los maxilares, que se caracteriza por un desarrollo, dolicocefálico y vertical de los maxila-

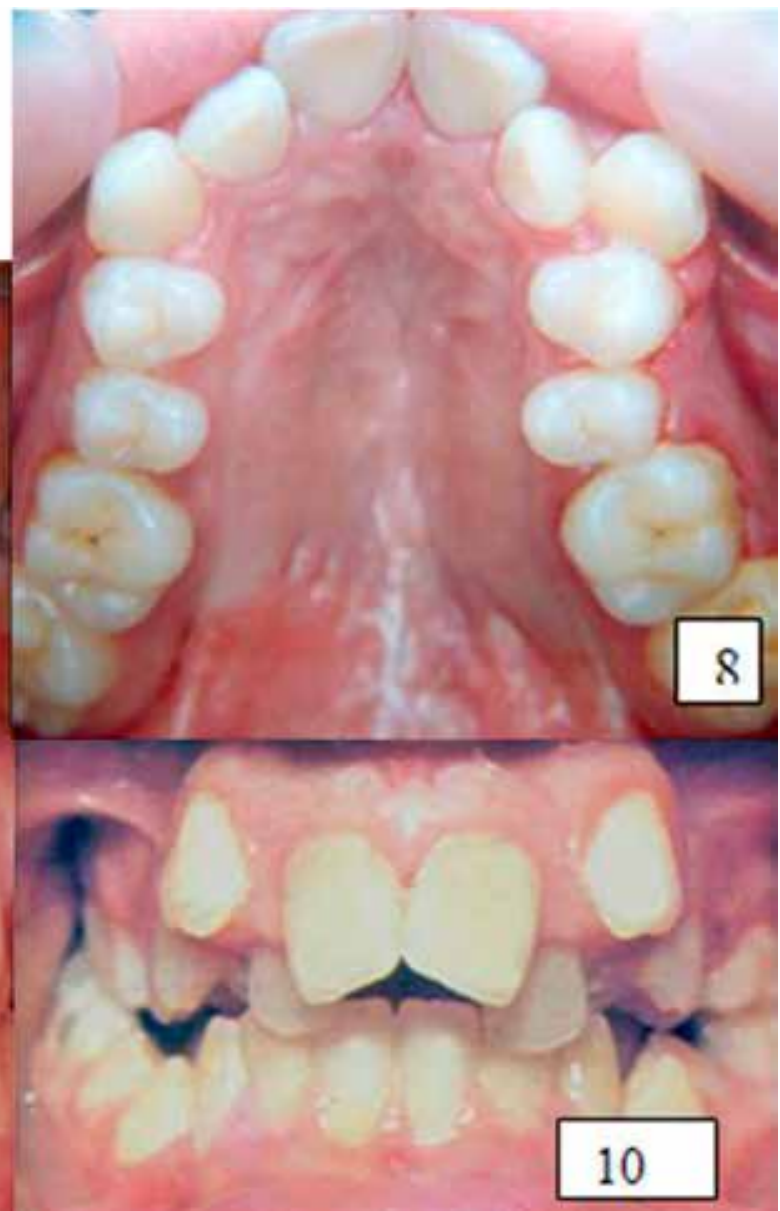


res con disminución del espacio disponible para las piezas dentarias permanentes, por lo que tendremos protrusión, apiñamiento dentario, piezas dentarias retenidas por la falta de espacio (Figura $4-8,9,10$ ).

Las alteraciones serán más severas y de mayor magnitud (Figura 5-6) en pacientes de mayor edad donde las deformaciones son más marcadas e irreversibles.

No siempre el paciente respirador bucal presentará las características mencionadas. Cuando la patología respiratoria se debe a obstrucciones de las vías respiratorias bajas por hipertrofia amigdalina (Figura I I-I2) y con el agregado de factores genéticos predisponentes, podemos observar, avance mandibular, por empuje lingual anterior, y si bien no es característica la respiración bucal permanente, el paciente debe abrir la boca en determinados momentos para compensar la dificultad en la entrada de aire. Se observa hipodesarrollo del maxilar superior, lo que ubica al labio superior retraído con el ángulo nasolabial aumentado, mordida invertida anterior y bilateral. Clase III de Angle (Figura I3-I4).

\section{Cambios de orden general}

\section{Alteraciones esqueléticas}

Cuando el problema respiratorio se debe a obstrucción de las vías respiratorias altas, el respirador bucal presentará alteraciones posturales que pueden ser leves o severas.

Al tener el paciente la boca abierta para poder respirar, mandíbula cambia el sistema de palanca y las fuerzas se desplazan pues cambian los puntos de apoyo ${ }^{7}$. Este desequilibrio, es compensado por cambios en la postura de la cabeza con respecto al cuerpo, marcada curvatura a nivel del cuello), generando un nuevo equilibrio patológico, ya que el hueso hioides está fijado solo por haces musculares a la apófisis estiloides, a la mandíbula, al omóplato, al esternón y a la clavícula. Las alteraciones posturales tendrán repercusión a lo largo de toda la columna vertebral e incluso hasta la planta de los pies. En este cambio postural se involucra además de la cabeza, que se coloca adelantada y hacia abajo, toda la columna vertebral, con marcada cifosis a nivel dorsal, hombros inclinados hacia delante. También, se puede observar escoliosis que se manifiesta con

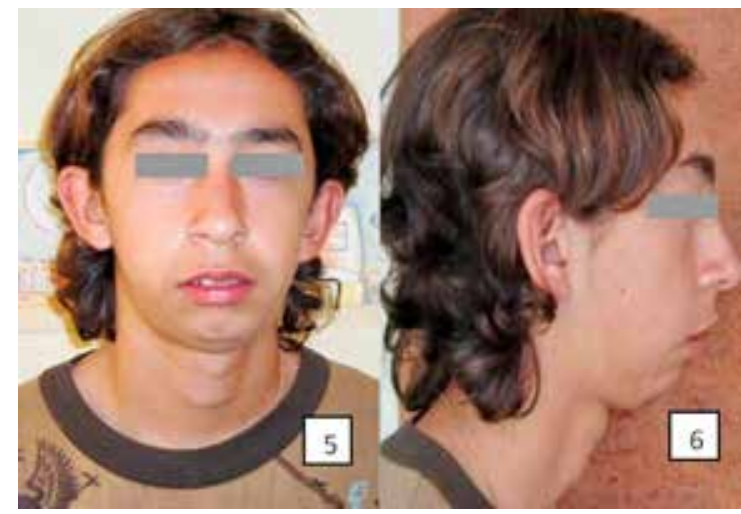

Figura 5-6

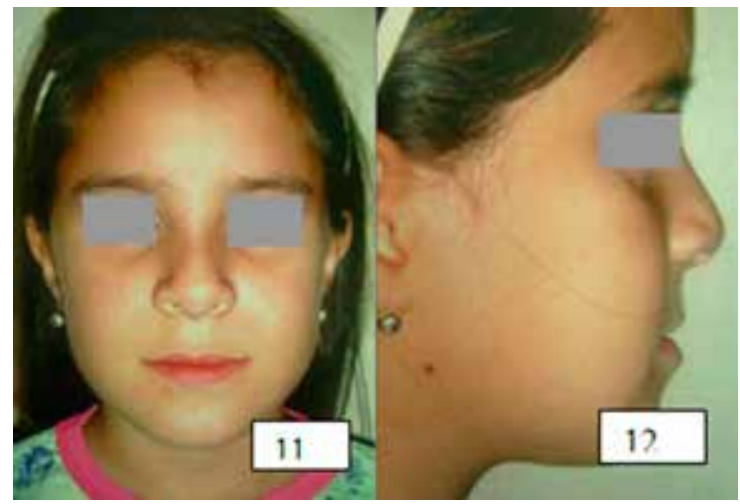

Figura II-I2

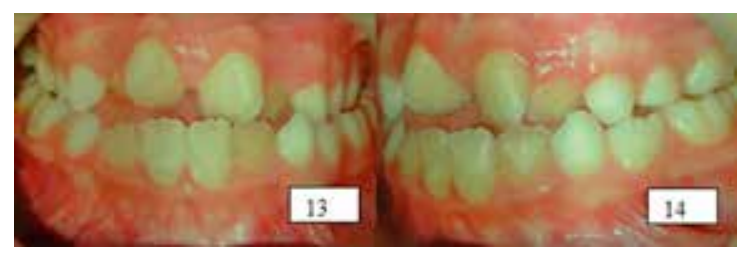

Figura 13-14
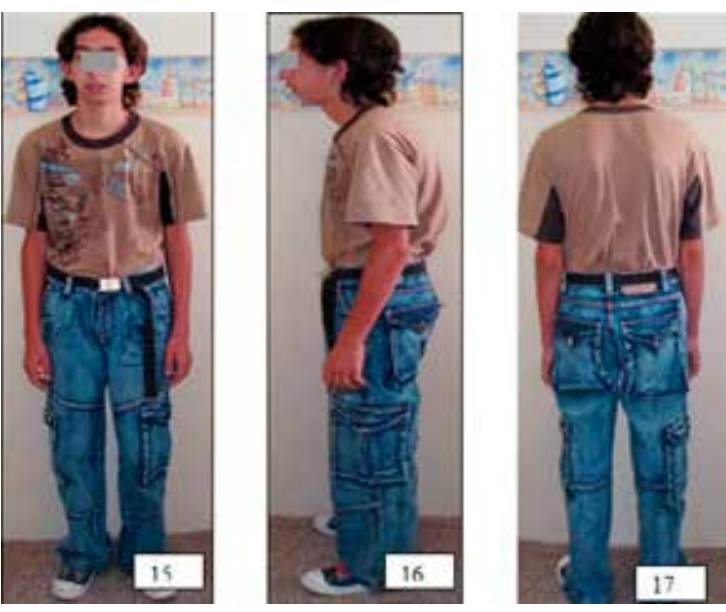

Figura $15-16-17$ 

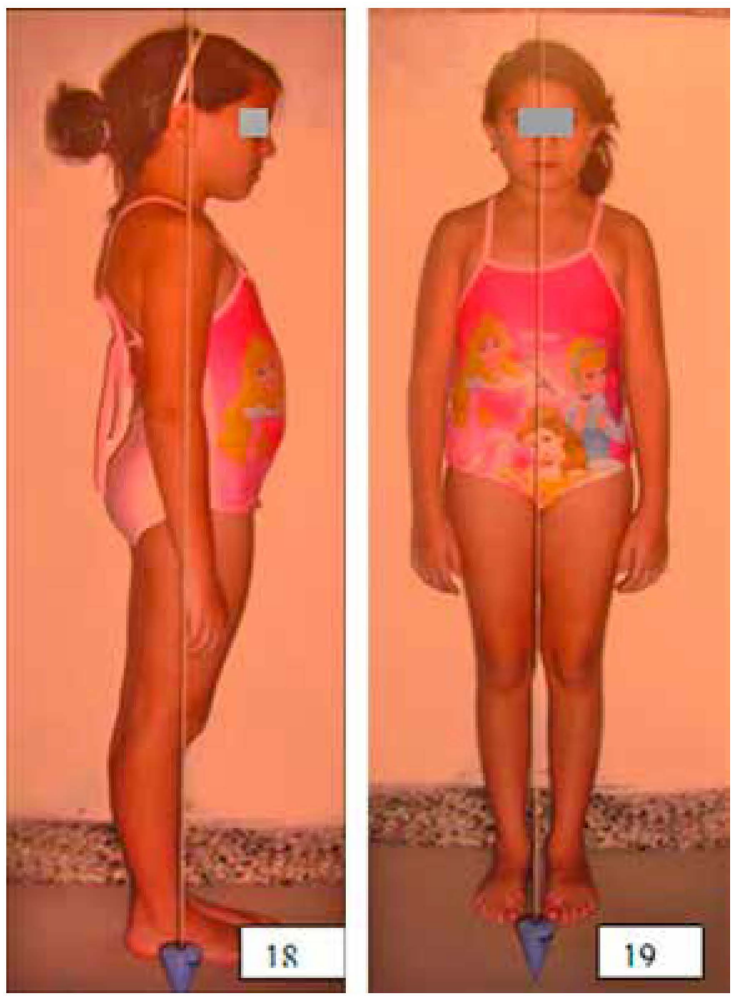

Figura 18-19

diferencia de altura de los hombros (Figura 15, 16 y (7).

En casos en que la respiración bucal se produzca por obstrucción de vías respiratorias bajas podremos observar, actitudes posturales con lordosis a nivel lumbar (Figura 18 y 19) ${ }^{7-10}$.

\section{Alteraciones fisiológicas}

Trastornos en la audición: Se presentan alteraciones en la membrana timpánica, con pérdida de la audición y con frecuentes otitis. El habla es imprecisa con problemas de articulación y sonoridad extraña, debido a la hiper o hiponasalidad. Disturbios alimentarios: La función masticatoria es ineficaz, por la incoordinación de la respiración con la masticación y la dificultad que ocasiona, alimentarse y respirar al mismo tiempo. Esto provoca problemas digestivos y atragantamiento. El respirador bucal puede ser obeso por comer grandes cantidades de alimentos, masticando poco, para tragar rápido y poder respirar; o también, puede ser delgado, cuando introduce, pequeñas cantidades de alimento, mastica mucho se cansa y come poco.

Trastornos en el sueño: Apnea del sueño, con interrupción momentánea de la respiración.
Sueño agitado, con despertar cansado y somnolencia. Ronquidos. Babeo nocturno. Pesadillas. El ronquido, producido por la vibración de los tejidos flexibles de la vía aérea superior que se estrecha durante el sueño, constituye siempre un signo de alarma que nos orienta a preguntar si el paciente presenta alteraciones de la función respíratoria ${ }^{8}$.

\section{Alteraciones psicosociales}

La disminución crónica de oxígeno, genera baja oxigenación cerebral. Esto se traduce en apatía, disminución de la actividad voluntaria, trastornos en la memoria, disminución de la capacidad para fijar la atención y cansancio crónico, asociado a los trastornos de sueño. Puede haber, trastornos en el comportamiento, ansiedad, irritabilidad e impulsividad. Se presentan trastornos en el lenguaje y la voz por la presencia de dislalias, esto sumado a las dificultades de concentración que se manifiesta con bajo rendimiento escolar?

\section{Conclusión}

Observar detalladamente al respirador bucal, revela una problemática amplia y variada, que afecta $\circ$ puede afectar partes muy distantes del individuo, desde las estructuras orofaciales a los pies. Si bien, no todos los cambios mencionados se presentan en un mismo paciente, la evaluación correcta del respirador bucal, nos permitirá el diagnostico y tratamiento precoz que es lo ideal, ya que las alteraciones serán mas graves y de mayor magnitud cuando reciba tratamiento tardío y existan predisposiciones genéticas para su desarrollo.

Sistematizar la observación y el análisis de los cambios encontrados permitirá, mejorar el diagnóstico y la terapia, buscando ayuda correspondiente en las distintas especialidades, realizando tratamiento interdisciplinario, optimizando la rehabilitación del paciente en forma integral. 


\section{Bibliografía}

I. Proffit W. Ortodoncia Contemporánea Etiología de los problemas Ortodóncicos. ed. España; 2008.

2. Fieramoscas F., Lezama E., Manrique R., Quiroz O., Farias $M$. La función respiratoria y su repercusión en el sistema estomatognático. [en linea]. Rev. Latinoamericana de Ortodoncia y Odontopediatría; 2007 [consulta 19 de junio de 2012 ] disponible en: www.ortodoncia.ws/publicacionesa/2007/art5.asp. ISSN: I3 17-5823.

3. Muñoz Martinez E., Xaviera Garcia. Fisiología células, órganos y sistemas: aparato respiratorio. México. Ed. Fondo de Cultura Económica; 1994. p. 29-72.

4. Preston B. Las vías respiratorias superiores y la morfología craneal. En: Graber T, Vanarsdall R, Vig W. Ortodoncia $4^{\circ}$ ed. España: Elseiver; 2006. p. II7-43.

5. Proffit WR., Fieds HW. Ortodoncia contemporánea. Teoría y práctica $3^{\circ}$ Ed. Madrid: Mosby;200I. p. 350-7.

6. Morera Perez A. Resultados de la Terapia Miofuncional en pacientes con incompetencia bilabial. Revista Ortodoncia Española. 2005; 45 (3): I76I91

7. Ricard F. Tratado de Osteopatía Craneal. Articulación Temporomandibular: Análisis y tratamiento ortodóncico. $2^{\mathrm{a}}$ Ed. Madrid: Médica Panamericana; 2005. p. 380-89.

8. Varela M. Ortodoncia y patología asociada al sueño. Ortodoncia Interdisciplinar. Madrid: Océano/ Ergon; 2.005. p. 724-748.

9. Salvador Perello A. Síndrome de apneas hipoapneas del sueño. Papel del Ortodoncista. Revista Ortodoncia Clínica. 2007; 10 (3): I38- 149.

10. Lima L. Postural alterations in children whithmouth breathingg assessed by computarized biophoto grammetry. J. Apl. Oral Sci 2004 I2 (3) 1232-7. 